\title{
Article \\ Applications of the $q$-Srivastava-Attiya Operator Involving a Certain Family of Bi-Univalent Functions Associated with the Horadam Polynomials
}

\author{
Hari Mohan Srivastava ${ }^{1,2,3,4}(\mathbb{D})$, Abbas Kareem Wanas 5 (D) and Rekha Srivastava $1, *(\mathbb{D})$ \\ 1 Department of Mathematics and Statistics, University of Victoria, Victoria, BC V8W 3R4, Canada; \\ harimsri@math.uvic.ca \\ 2 Department of Medical Research, China Medical University Hospital, China Medical University, \\ Taichung 40402, Taiwan \\ 3 Department of Mathematics and Informatics, Azerbaijan University, 71 Jeyhun Hajibeyli Street, \\ Baku AZ1007, Azerbaijan \\ 4 Section of Mathematics, International Telematic University Uninettuno, I-00186 Rome, Italy \\ 5 Department of Mathematics, College of Science, University of Al-Qadisiyah, Al Diwaniyah, \\ Al-Qadisiyah 54004, Iraq; abbas.kareem.w@qu.edu.iq \\ * Correspondence: rekhas@math.uvic.ca
}

check for updates

Citation: Srivastava, H.M.; Wanas, A.K.; Srivastava, R. Applications of the $q$-Srivastava-Attiya Operator Involving a Certain Family of Bi-Univalent Functions Associated with the Horadam Polynomials. Symmetry 2021, 13, 1230. https:// doi.org/10.3390/sym13071230

Academic Editors: Aviv Gibali and Markus Meringer

Received: 1 June 2021

Accepted: 26 June 2021

Published: 8 July 2021

Publisher's Note: MDPI stays neutral with regard to jurisdictional claims in published maps and institutional affiliations.

Copyright: (C) 2021 by the authors. Licensee MDPI, Basel, Switzerland. This article is an open access article distributed under the terms and conditions of the Creative Commons Attribution (CC BY) license (https:// creativecommons.org/licenses/by/ $4.0 /)$.

\begin{abstract}
In this article, by making use of the $q$-Srivastava-Attiya operator, we introduce and investigate a new family $\mathcal{S} \mathcal{W}_{\Sigma}(\delta, \gamma, \lambda, \mathfrak{s}, \mathrm{t}, q, r)$ of normalized holomorphic and bi-univalent functions in the open unit disk $\mathbb{U}$, which are associated with the Bazilevič functions and the $\lambda$-pseudo-starlike functions as well as the Horadam polynomials. We estimate the second and the third coefficients in the Taylor-Maclaurin expansions of functions belonging to the holomorphic and bi-univalent function class, which we introduce here. Furthermore, we establish the Fekete-Szegö inequality for functions in the family $\mathcal{S} \mathcal{W}_{\Sigma}(\delta, \gamma, \lambda, \mathfrak{s}, \mathrm{t}, q, r)$. Relevant connections of some of the special cases of the main results with those in several earlier works are also pointed out. Our usage here of the basic or quantum (or q-) extension of the familiar Hurwitz-Lerch zeta function $\Phi(z, s, a)$ is justified by the fact that several members of this family of zeta functions possess properties with local or non-local symmetries. Our study of the applications of such quantum (or $q$-) extensions in this paper is also motivated by the symmetric nature of quantum calculus itself.
\end{abstract}

Keywords: holomorphic functions; univalent functions; bi-univalent functions; Hurwitz-Lerch zeta function; Srivastava-Attiya operator; Bazilevič functions; $\lambda$-pseudo-starlike functions; Horadam polynomials; Taylor-Maclaurin expansions; coefficient estimates; Fekete-Szegö problem; subordination between holomorphic functions; $q$-Srivastava-Attiya operator; Hadamard product (or convolution)

MSC: Primary 30C45; Secondary 30C50; 33C05

\section{Introduction and Preliminaries}

We indicate by $\mathcal{A}$ the collection of functions, which are holomorphic in the open unit disk $\mathbb{U}$ given by

$$
\mathbb{U}=\{z: z \in \mathbb{C} \text { and }|z|<1\}
$$

and have the following normalized form:

$$
f(z)=z+\sum_{n=2}^{\infty} a_{n} z^{n} .
$$


We denote by $\mathcal{S}$ the sub-collection of the set $\mathcal{A}$ consisting of functions, which are also univalent in $\mathbb{U}$. According to the Koebe one-quarter theorem [1], every function $f \in \mathcal{S}$ has an inverse $f^{-1}$ defined by

$$
f^{-1}(f(z))=z \quad(z \in \mathbb{U})
$$

and

$$
f\left(f^{-1}(w)\right)=w \quad\left(|w|<r_{0}(f) ; r_{0}(f) \geqq \frac{1}{4}\right)
$$

where

$$
g(w)=f^{-1}(w)=w-a_{2} w^{2}+\left(2 a_{2}^{2}-a_{3}\right) w^{3}-\left(5 a_{2}^{3}-5 a_{2} a_{3}+a_{4}\right) w^{4}+\cdots .
$$

We say that a function $f \in \mathcal{A}$ is bi-univalent in $\mathbb{U}$ if both $f$ and it inverse $f^{-1}$ are univalent in $\mathbb{U}$. Let $\Sigma$ stand for the family of bi-univalent functions in $\mathbb{U}$ given by (1). Beginning with the pioneering work [2] on the subject by Srivastava et al. [2], a large number of works related to the subject have been (and continue to be) published (see, for example, Refs. [3-7]). From the work of Srivastava et al. [2], we choose to recall the following examples of functions in the family $\Sigma$ :

$$
\frac{z}{1-z}, \quad-\log (1-z) \quad \text { and } \quad \frac{1}{2} \log \left(\frac{1+z}{1-z}\right) .
$$

We notice that the family $\Sigma$ is not empty. However, the Koebe function is not a member of $\Sigma$. The problem to find the general coefficient bounds on the Taylor-Maclaurin coefficients

$$
\left|a_{n}\right| \quad(n \in \mathbb{N} ; n \geqq 3)
$$

for functions $f \in \Sigma$ is still not completely addressed for many of the subfamilies of the bi-univalent function family $\Sigma$.

Finding an upper bound for the functional $\left|a_{3}-\mu a_{2}^{2}\right|(f \in \mathcal{S})$ constitutes the FeketeSzegö type inequality (or problem) (see [8]). It originates from their disproof of the Littlewood-Paley conjecture that the coefficients of odd univalent functions are bounded by 1 . For some recent developments and examples, see $[9,10]$.

A function $f \in \mathcal{A}$ is called a Bazilevič function in $\mathbb{U}$ if the following inequality holds true (see [11]):

$$
\Re\left(\frac{z^{1-\gamma} f^{\prime}(z)}{(f(z))^{1-\gamma}}\right)>0 \quad(z \in \mathbb{U} ; \gamma \geqq 0) .
$$

On the other hand, a function $f \in \mathcal{A}$ is called a $\lambda$-pseudo-starlike function in $\mathbb{U}$ if the following inequality holds true (see [12]):

$$
\Re\left(\frac{z\left(f^{\prime}(z)\right)^{\lambda}}{f(z)}\right)>0 \quad(z \in \mathbb{U} ; \lambda \geqq 1) .
$$

Next, we recall the definition of subordination between holomorphic functions. For two functions $f, g \in \mathcal{A}$, we say that the function $f$ is subordinate to $g$, if there exists a Schwarz function $\omega$, which is holomorphic in $\mathbb{U}$ with the following property:

$$
\omega(0)=0 \quad \text { and } \quad|\omega(z)|<1 \quad(z \in \mathbb{U}),
$$

such that

$$
f(z)=g(\omega(z)) .
$$

This subordination is symbolically written as follows:

$$
f \prec g \quad \text { or } \quad f(z) \prec g(z) \quad(z \in \mathbb{U}) .
$$


It is well known that if the function $g$ is univalent in $\mathbb{U}$, then the following equivalence holds true (see [13]):

$$
f \prec g \quad(z \in \mathbb{U}) \Longleftrightarrow f(0)=g(0) \text { and } f(\mathbb{U}) \subseteq g(\mathbb{U}) .
$$

Jackson $[14,15]$ introduced the $q$-derivative operator $\mathfrak{D}_{q}$ of a function $f$ as follows:

$$
\mathfrak{D}_{q} f(z)=\frac{f(z)-f(q z)}{(1-q) z} \quad(0<q<1 ; z \neq 0) .
$$

The following limit relationship is clear:

$$
\lim _{q \rightarrow 1-} \mathfrak{D}_{q} f(z)=f^{\prime}(z) \quad \text { and } \quad \mathfrak{D}_{q} f(0)=f^{\prime}(0) .
$$

For more conceptual details on the $q$-derivative operator $\mathfrak{D}_{q}$, see [16-18].

For a function $f \in \mathcal{A}$ defined by (1), we deduce the following result:

$$
\mathfrak{D}_{q} f(z)=1+\sum_{n=2}^{\infty}[n]_{q} a_{n} z^{n-1}
$$

where $[n]_{q}$, called the $q$-analogue of $n \in \mathbb{N}$, is given by

$$
[n]_{q}=\frac{1-q^{n}}{1-q} \quad(n \in \mathbb{N} \backslash\{1\}),
$$

$\mathbb{N}$ being the set of positive integers.

As $q \longrightarrow 1-$, we have $[n]_{q} \longrightarrow n$ and $[0]_{q}=0$.

The widely and extensively studied Srivastava-Attiya operator was defined by Srivastava and Attiya [19] by using the Hurwitz-Lerch zeta function $\Phi(z, s, a)$ which is systematically discussed in the recent survey articles [20,21]. For details about the relationships of the function $\Phi(z, s, a)$ with several important functions of the analytic number theory, the interested reader can refer to Chapter I in [22]).

Shah and Noor [23] (see also [24]) studied the following $q$-analogue of the HurwitzLerch zeta function $\Phi(z, s, a)$ :

$$
\phi_{q}(\mathfrak{s}, \mathfrak{t} ; z)=\sum_{n=0}^{\infty} \frac{z^{n}}{[n+\mathfrak{t}]_{q}^{\mathfrak{s}}}
$$

where $\mathfrak{t} \in \mathbb{C} \backslash \mathbb{Z}_{0}^{-}, \mathfrak{s} \in \mathbb{C}$ when $|z|<1$ and $\Re(\mathfrak{s})>1$ when $|z|=1$. The normalized form of the series (3) is defined by

$$
\psi_{q}(\mathfrak{s}, \mathfrak{t} ; z)=[1+\mathfrak{t}]_{q}^{\mathfrak{s}}\left(\phi_{q}(\mathfrak{s}, \mathfrak{t} ; z)-[\mathfrak{t}]_{q}^{-\mathfrak{s}}\right)=z+\sum_{n=2}^{\infty}\left(\frac{[1+\mathfrak{t}]_{q}}{[n+\mathfrak{t}]_{q}}\right)^{\mathfrak{s}} z^{n}
$$

By using (1) and (4), Shah and Noor [23] defined the $q$-Srivastava-Attiya operator $\mathfrak{J}_{q, \mathfrak{t}}^{\mathfrak{s}} f: \mathcal{A} \longrightarrow \mathcal{A}$ as follows:

Definition 1 (see [23]; see also [24]). The q-Srivastava-Attiya operator $\mathfrak{J}_{q, t}^{\mathfrak{s}} f: \mathcal{A} \longrightarrow \mathcal{A}$ is defined by

$$
\mathfrak{J}_{q, \mathfrak{t}}^{\mathfrak{s}} f(z)=\psi_{q}(\mathfrak{s}, \mathfrak{t} ; z) * f(z)=z+\sum_{n=2}^{\infty}\left(\frac{[1+\mathfrak{t}]_{q}}{[n+\mathfrak{t}]_{q}}\right)^{\mathfrak{s}} a_{n} z^{n},
$$

where the symbol $*$ stands for the Hadamard product (or convolution). 
In recent years, several authors studied many applications of $q$-calculus associated with various families of holomorphic and univalent (or multivalent) functions (see, for example, [10,25-33]).

In his recently-published survey-cum-expository review article, Srivastava [34] explored the mathematical applications of $q$-calculus, fractional $q$-calculus and the fractional $q$-derivative operators in Geometric Function Theory of Complex Analysis. Srivastava [34] also exposed the not-yet-widely-understood fact that the so-called $(p, q)$-variation of classical $q$-calculus is a rather trivial and inconsequential variation of classical $q$-calculus, the additional parameter $p$ being redundant or superfluous (see, for details, [34], p. 340).

Here, in this paper, we made use of the basic or quantum (or $q$-) extension $\phi_{q}(\mathfrak{s}, \mathfrak{t} ; z)$ which, when $q \rightarrow 1-$, yields the familiar Hurwitz-Lerch zeta function $\Phi(z, \mathfrak{s}, \mathfrak{t})$. Just as we pointed out above, local or non-local symmetries are known to exist in some properties of several members of the family of the Hurwitz-Lerch zeta functions. Further motivation for our study of the applications of such quantum (or $q$-) extensions in this paper can be found in the book chapter entitled Symmetric Quantum Calculus, in [35].

Remark 1. The operator $\mathfrak{J}_{q, \mathfrak{t}}^{\mathfrak{s}}$ is a generalization of several known operators studied in earlier investigations, which are recalled below.

1. For $q \longrightarrow 1-$, the function $\phi_{q}(\mathfrak{s}, \mathfrak{t} ; z)$ reduces to the Hurwitz-Lerch zeta function $($ see $[20,21])$ and the operator $\mathfrak{J}_{q, \mathfrak{t}}^{\mathfrak{s}}$ coincides with the Srivastava-Attiya operator in [19]. Various applications of the Srivastava-Attiya operator are found in [36-38] and in the references cited in each of these earlier works.

2. For $\mathfrak{s}=1$, the operator $\mathfrak{J}_{\mathfrak{q}, \mathfrak{t}}^{\mathfrak{s}}$ reduces to the $q$-Bernardi operator (see [39]).

3. For $\mathfrak{s}=\mathfrak{t}=1$, the operator $\mathfrak{J}_{q, \mathfrak{t}}^{\mathfrak{s}}$ reduces to the $q$-Libera operator (see [39]).

4. For $q \longrightarrow 1-$ and $\mathfrak{s}=1$, the operator $\mathfrak{J}_{q, \mathfrak{t}}^{\mathfrak{s}}$ reduces to the Bernardi operator (see [40]).

5. For $q \longrightarrow 1-, \mathfrak{s}=1$ and $\mathfrak{t}=0$, the operator $\mathfrak{J}_{\mathfrak{q}, \mathfrak{t}}^{\mathfrak{s}}$ reduces to the Alexander operator (see [41]).

Recently, Hörçum and Koçer [42] considered the familiar Horadam polynomials $h_{n}(r)$, which are given by Definition 2 below, from Geometric Function Theory of Complex Analysis.

Definition 2 (see [42,43]). The Horadam polynomials $h_{n}(r)$ are given by the following recurrence relation:

$$
h_{n}(r)=\mathfrak{p} r h_{n-1}(r)+\mathfrak{q} h_{n-2}(r) \quad(r \in \mathbb{R} ; n \in \mathbb{N}=\{1,2,3, \cdots\})
$$

with

$$
h_{1}(r)=a \text { and } h_{2}(r)=b r
$$

for some real constants $a, b, \mathfrak{p}$ and $\mathfrak{q}$. Moreover, the characteristic equation of the recurrence relation (5) is given by

$$
t^{2}-\mathfrak{p} r t-\mathfrak{q}=0
$$

which has the following two real roots:

$$
\alpha=\frac{\mathfrak{p} r+\sqrt{\mathfrak{p}^{2} r^{2}+4 \mathfrak{q}}}{2} \quad \text { and } \quad \beta=\frac{\mathfrak{p} r-\sqrt{\mathfrak{p}^{2} r^{2}+4 \mathfrak{q}}}{2} .
$$

Remark 2. We record here some special cases of the Horadam polynomials $h_{n}(r)$ by appropriately choosing the parameters $a, b, \mathfrak{p}$ and $\mathfrak{q}$.

1. Taking $a=b=\mathfrak{p}=\mathfrak{q}=1$, we obtain the Fibonacci polynomials $F_{n}(r)$.

2. Taking $a=2$ and $b=\mathfrak{p}=\mathfrak{q}=1$, we get the Lucas polynomials $L_{n}(r)$.

3. Taking $a=\mathfrak{q}=1$ and $b=\mathfrak{p}=2$, we have the Pell polynomials $P_{n}(r)$.

4. Taking $a=b=\mathfrak{p}=2$ and $\mathfrak{q}=1$, we find the Pell-Lucas polynomials $Q_{n}(r)$.

5. Taking $a=b=1, \mathfrak{p}=2$ and $\mathfrak{q}=-1$, we obtain the Chebyshev polynomials $T_{n}(r)$ of the first kind. 
6. Taking $a=1, b=\mathfrak{p}=2$ and $\mathfrak{q}=-1$, we have the Chebyshev polynomials $U_{n}(r)$ of the second kind.

For widespread usages and applications of various families of orthogonal polynomials and other special functions and specific polynomials, see [43-46].

The Horadam polynomials $h_{n}(r)$ are generated by (see [42]):

$$
\Pi(r, z)=\sum_{n=1}^{\infty} h_{n}(r) z^{n-1}=\frac{a+(b-a \mathfrak{p}) r z}{1-\mathfrak{p} r z-\mathfrak{q} z^{2}} .
$$

The Horadam polynomials $h_{n}(r)$ were recently applied in a similar context by Srivastava et al. [47]. It was followed by many sequels to [47] (see, for example, [48-54]).

Remark 3. The motivation of our present investigation stems, at least in part, from the need for the upper bounds of the Taylor-Maclaurin coefficients of normalized functions belonging to various subclasses of analytic and univalent (or multivalent) functions in the open unit disk $\mathbb{U}$. The proof of the celebrated 68-year-old Bieberbach conjecture, which is attributed to Ludwig Bieberbach (18861982), by Louis de Branges in the year 1984 has indeed provided impetus to studies on coefficient estimate problems as well as on Fekete-Szegö type coefficient inequalities in recent years.

\section{A Set of Main Results}

We begin this section by defining the new family $\mathcal{S} \mathcal{W}_{\Sigma}(\delta, \gamma, \lambda, \mathfrak{s}, \mathfrak{t}, q, r)$.

Definition 3. For $0 \leqq \delta \leqq 1, \gamma \geqq 0, \lambda \geqq 1$ and $r \in \mathbb{R}$, a function $f \in \Sigma$ is said to be in the family $\mathcal{S W}_{\Sigma}(\delta, \gamma, \lambda, \mathfrak{s}, \mathfrak{t}, q, r)$ if it fulfills the following subordination conditions:

$$
(1-\delta) \frac{z^{1-\gamma}\left(\mathfrak{J}_{q, t}^{\mathfrak{s}} f(z)\right)^{\prime}}{\left(\mathfrak{J}_{q, t}^{\mathfrak{s}} f(z)\right)^{1-\gamma}}+\delta \frac{z\left[\left(\mathfrak{J}_{q, t}^{\mathfrak{s}} f(z)\right)^{\prime}\right]^{\lambda}}{\mathfrak{J}_{q, t}^{\mathfrak{s}} f(z)} \prec \Pi(r, z)+1-a
$$

and

$$
(1-\delta) \frac{w^{1-\gamma}\left(\mathfrak{J}_{q, t}^{\mathfrak{s}} g(w)\right)^{\prime}}{\left(\mathfrak{J}_{q, t}^{\mathfrak{s}} g(w)\right)^{1-\gamma}}+\delta \frac{w\left[\left(\mathfrak{J}_{q, t}^{\mathfrak{s}} g(w)\right)^{\prime}\right]^{\lambda}}{\mathfrak{J}_{q, t}^{\mathfrak{s}} g(w)} \prec \Pi(r, w)+1-a,
$$

where $a$ is real constant and the function $g=f^{-1}$ is given by (2).

Remark 4. For brevity and convenience, the notation $\mathcal{S W}_{\Sigma}(\delta, \gamma, \lambda, \mathfrak{s , t}, q, r)$ for the holomorphic and bi-univalent function class, which we introduced in Definition 3 above, does not include the parameters $a, b, \mathfrak{p}$ and $\mathfrak{q}$ involved in Definition 1 of the Horadam polynomials $h_{n}(r)$. In fact, the role of each of these notationally left-out parameters $a, b, \mathfrak{p}$ and $\mathfrak{q}$, which is detailed above in Remark 2 , is to relate the Horadam polynomials $h_{n}(r)$ with many simpler polynomial systems (see also Remark 5 below).

Our first main result is asserted by Theorem 1 below.

Theorem 1. For $0 \leqq \delta \leqq 1, \gamma \geqq 0, \lambda \geqq 1$ and $r \in \mathbb{R}$, let $f \in \mathcal{A}$ be in the family $\mathcal{S W}_{\Sigma}(\delta, \gamma, \lambda, \mathfrak{s}, \mathfrak{t}, q, r)$. Then

$$
\left|a_{2}\right| \leqq \frac{|b r|[2+\mathfrak{t}]_{\mathfrak{q}}^{\mathfrak{s}} \sqrt{|b r|[3+\mathfrak{t}]_{\mathfrak{q}}^{\mathfrak{s}}}}{\Lambda(\delta, \gamma, \lambda, \mathfrak{s}, \mathfrak{t}, q ; b . r)}
$$




$$
\begin{aligned}
& (\Lambda(\delta, \gamma, \lambda, \mathfrak{s}, \mathfrak{t}, q ; b . r) \\
& \left.:=\sqrt{\left|[(\Omega(\delta, \gamma, \lambda, \mathfrak{s}, \mathfrak{t}, q)+\Gamma(\delta, \gamma, \lambda, \mathfrak{s}, \mathfrak{t}, q)) b-\mathfrak{p Y}(\delta, \gamma, \lambda, \mathfrak{s}, \mathfrak{t}, q)] b r^{2}-\mathfrak{q} a Y(\delta, \gamma, \lambda, \mathfrak{s}, \mathfrak{t}, q)\right|}\right)
\end{aligned}
$$

and

$$
\begin{aligned}
&\left|a_{3}\right| \leqq \frac{|b r|[3+\mathfrak{t}]_{q}^{\mathfrak{s}}}{[(1-\delta)(\gamma+2)+\delta(3 \lambda-1)] \cdot[1+\mathfrak{t}]_{\mathfrak{q}}^{\mathfrak{s}}} \\
& \quad+\frac{b^{2} r^{2}[2+\mathfrak{t}]_{q}^{2 \mathfrak{s}}}{[(1-\delta)(\gamma+1)+\delta(2 \lambda-1)]^{2} \cdot[1+\mathfrak{t}]_{\mathfrak{q}}^{2 \mathfrak{s}}}
\end{aligned}
$$

where

$$
\begin{gathered}
\Omega(\delta, \gamma, \lambda, \mathfrak{s}, \mathfrak{t}, q)=[(1-\delta)(\gamma+2)+\delta(3 \lambda-1)][1+\mathfrak{t}]_{q}^{\mathfrak{s}}[2+\mathfrak{t}]_{q}^{2 \mathfrak{s}} \\
\Gamma(\delta, \gamma, \lambda, \mathfrak{s}, \mathfrak{t}, q)=\left[\frac{1}{2}(1-\delta)(\gamma+2)(\gamma-1)+\delta(2 \lambda(\lambda-2)+1)\right][3+\mathfrak{t}]_{q}^{\mathfrak{s}}[1+\mathfrak{t}]_{q}^{2 \mathfrak{s}}
\end{gathered}
$$

and

$$
\mathrm{Y}(\delta, \gamma, \lambda, \mathfrak{s}, \mathfrak{t}, q)=[(1-\delta)(\gamma+1)+\delta(2 \lambda-1)]^{2}[3+\mathfrak{t}]_{q}^{\mathfrak{s}}[1+\mathfrak{t}]_{q}^{2 \mathfrak{s}}
$$

Proof. Let $f \in \mathcal{S} \mathcal{W}_{\Sigma}(\delta, \gamma, \lambda, \mathfrak{s}, \mathfrak{t}, q, r)$. Then there are two holomorphic functions $u, v$ : $\mathbb{U} \longrightarrow \mathbb{U}$ given by

$$
u(z)=u_{1} z+u_{2} z^{2}+u_{3} z^{3}+\cdots \quad(z \in \mathbb{U})
$$

and

$$
v(w)=v_{1} w+v_{2} w^{2}+v_{3} w^{3}+\cdots \quad(w \in \mathbb{U})
$$

with

$$
u(0)=v(0)=0 \quad \text { and } \quad \max \{|u(z)|,|v(w)|\}<1 \quad(z, w \in \mathbb{U}),
$$

such that

$$
(1-\delta) \frac{z^{1-\gamma}\left(\mathfrak{J}_{q, \mathrm{t}}^{\mathfrak{s}} f(z)\right)^{\prime}}{\left(\mathfrak{J}_{\mathfrak{q}, \mathrm{t}}^{\mathfrak{s}} f(z)\right)^{1-\gamma}}+\delta \frac{z\left[\left(\mathfrak{J}_{q, \mathfrak{t}}^{\mathfrak{s}} f(z)\right)^{\prime}\right]^{\lambda}}{\mathfrak{J}_{q, \mathrm{t}}^{\mathfrak{s}} f(z)}=\Pi(r, u(z))-a
$$

and

$$
(1-\delta) \frac{w^{1-\gamma}\left(\mathfrak{J}_{q, \mathfrak{t}}^{\mathfrak{s}} g(w)\right)^{\prime}}{\left(\mathfrak{J}_{q, \mathfrak{t}}^{\mathfrak{s}} g(w)\right)^{1-\gamma}}+\delta \frac{w\left[\left(\mathfrak{J}_{q, \mathrm{t}}^{\mathfrak{s} g} g(w)\right)^{\prime}\right]^{\lambda}}{\mathfrak{J}_{q, \mathrm{t}}^{\mathfrak{s}} g(w)}=\Pi(r, v(w))-a
$$

or, equivalently,

$$
\begin{gathered}
(1-\delta) \frac{z^{1-\gamma}\left(\mathfrak{J}_{q, \mathrm{t}}^{\mathfrak{s}} f(z)\right)^{\prime}}{\left(\mathfrak{J}_{\mathfrak{q}, \mathrm{t}}^{\mathfrak{s}} f(z)\right)^{1-\gamma}}+\delta \frac{z\left[\left(\mathfrak{J}_{q, \mathrm{t}}^{\mathfrak{s}} f(z)\right)^{\prime}\right]^{\lambda}}{\mathfrak{J}_{q, \mathrm{t}}^{\mathfrak{s}} f(z)} \\
\quad=1+h_{1}(r)+h_{2}(r) u(z)+h_{3}(r) u^{2}(z)+\cdots
\end{gathered}
$$


and

$$
\begin{aligned}
(1-\delta) \frac{w^{1-\gamma}\left(\mathfrak{J}_{q, t}^{\mathfrak{s}} g(w)\right)^{\prime}}{\left(\mathfrak{J}_{\mathfrak{q}, t}^{\mathfrak{s}} g(w)\right)^{1-\gamma}}+\delta \frac{w\left[\left(\mathfrak{J}_{q, t}^{\mathfrak{s}}, g(w)\right)^{\prime}\right]^{\lambda}}{\mathfrak{J}_{\mathfrak{q}, \mathrm{t}}^{\mathfrak{s}} g(w)} \\
\quad=1+h_{1}(r)+h_{2}(r) v(w)+h_{3}(r) v^{2}(w)+\cdots .
\end{aligned}
$$

Combining (10)-(13) yields the following relation:

$$
\begin{aligned}
& (1-\delta) \frac{z^{1-\gamma}\left(\mathfrak{J}_{q, t}^{\mathfrak{s}} f(z)\right)^{\prime}}{\left(\mathfrak{J}_{q, t}^{\mathfrak{s}} f(z)\right)^{1-\gamma}}+\delta \frac{z\left[\left(\mathfrak{J}_{q, t}^{\mathfrak{s}} f(z)\right)^{\prime}\right]^{\lambda}}{\mathfrak{J}_{q, t}^{\mathfrak{s}} f(z)} \\
& =1+h_{2}(r) u_{1} z+\left[h_{2}(r) u_{2}+h_{3}(r) u_{1}^{2}\right] z^{2}+\cdots
\end{aligned}
$$

and

$$
\begin{aligned}
(1-\delta) \frac{w^{1-\gamma}\left(\mathfrak{J}_{q, t}^{\mathfrak{s}} g(w)\right)^{\prime}}{\left(\mathfrak{J}_{q, t}^{\mathfrak{s}} g(w)\right)^{1-\gamma}}+\delta \frac{w\left[\left(\mathfrak{J}_{q, t}^{\mathfrak{s}} g(w)\right)^{\prime}\right]^{\lambda}}{\mathfrak{J}_{q, t}^{\mathfrak{s}} g(w)} \\
\quad=1+h_{2}(r) v_{1} w+\left[h_{2}(r) v_{2}+h_{3}(r) v_{1}^{2}\right] w^{2}+\cdots
\end{aligned}
$$

It is known that, if

$$
\max \{|u(z)|,|v(w)|\}<1 \quad(z, w \in \mathbb{U}),
$$

then

$$
\left|u_{j}\right| \leqq 1 \quad \text { and } \quad\left|v_{j}\right| \leqq 1 \quad(\forall j \in \mathbb{N}) .
$$

Now, by comparing the corresponding coefficients in (14) and (15), we find that

$$
\begin{gathered}
\frac{[(1-\delta)(\gamma+1)+\delta(2 \lambda-1)][1+\mathfrak{t}]_{q}^{\mathfrak{g}}}{[2+\mathfrak{t}]_{q}^{\mathfrak{s}}} a_{2}=h_{2}(r) u_{1}, \\
\frac{[(1-\delta)(\gamma+2)+\delta(3 \lambda-1)][1+\mathfrak{t}]_{q}^{\mathfrak{s}}}{[3+\mathfrak{t}]_{q}^{\mathfrak{s}}} a_{3} \\
+\frac{\left[\frac{1}{2}(1-\delta)(\gamma+2)(\gamma-1)+\delta(2 \lambda(\lambda-2)+1)\right][1+\mathfrak{t}]_{q}^{2 \mathfrak{s}}}{[2+\mathfrak{t}]_{q}^{2 \mathfrak{s}}} a_{2}^{2} \\
=h_{2}(r) u_{2}+h_{3}(r) u_{1}^{2}, \\
-\frac{[(1-\delta)(\gamma+1)+\delta(2 \lambda-1)][1+\mathfrak{t}]_{q}^{\mathfrak{s}}}{[2+\mathfrak{t}]_{q}^{\mathfrak{s}}} a_{2}=h_{2}(r) v_{1}
\end{gathered}
$$

and

$$
\begin{gathered}
\frac{[(1-\delta)(\gamma+2)+\delta(3 \lambda-1)][1+\mathfrak{t}]_{\mathfrak{q}}^{\mathfrak{s}}}{[3+\mathfrak{t}]_{\mathfrak{q}}^{\mathfrak{s}}}\left(2 a_{2}^{2}-a_{3}\right) \\
+\frac{\left[\frac{1}{2}(1-\delta)(\gamma+2)(\gamma-1)+\delta(2 \lambda(\lambda-2)+1)\right][1+\mathfrak{t}]_{\mathfrak{q}}^{2 \mathfrak{s}}}{[2+\mathfrak{t}]_{q}^{2 \mathfrak{s}}} a_{2}^{2} \\
=h_{2}(r) v_{2}+h_{3}(r) v_{1}^{2} .
\end{gathered}
$$


It follows from (17) and (19) that

$$
u_{1}=-v_{1}
$$

and

$$
\frac{2[(1-\delta)(\gamma+1)+\delta(2 \lambda-1)]^{2}[1+\mathfrak{t}]_{q}^{2 \mathfrak{s}}}{[2+\mathfrak{t}]_{q}^{2 \mathfrak{s}}} a_{2}^{2}=h_{2}^{2}(r)\left(u_{1}^{2}+v_{1}^{2}\right) .
$$

If we add (18) to (20), we find that

$$
\begin{gathered}
\left(\frac{2[(1-\delta)(\gamma+2)+\delta(3 \lambda-1)][1+\mathfrak{t}]_{q}^{\mathfrak{s}}}{[3+\mathfrak{t}]_{q}^{\mathfrak{s}}}\right. \\
\left.\quad+\frac{2\left[\frac{1}{2}(1-\delta)(\gamma+2)(\gamma-1)+\delta(2 \lambda(\lambda-2)+1)\right][1+\mathfrak{t}]_{q}^{2 \mathfrak{s}}}{[2+\mathfrak{t}]_{q}^{2 \mathfrak{s}}}\right) a_{2}^{2} \\
\quad=h_{2}(r)\left(u_{2}+v_{2}\right)+h_{3}(r)\left(u_{1}^{2}+v_{1}^{2}\right) .
\end{gathered}
$$

Upon substituting the value of $u_{1}^{2}+v_{1}^{2}$ from (22) into the right-hand side of (23), we deduce the following result:

$$
a_{2}^{2}=\frac{h_{2}^{3}(r)[3+\mathfrak{t}]_{q}^{\mathfrak{s}}[2+\mathfrak{t}]_{q}^{2 \mathfrak{s}}\left(u_{2}+v_{2}\right)}{2\left[h_{2}^{2}(r)(\Omega(\delta, \gamma, \lambda, \mathfrak{s}, \mathfrak{t}, q)+\Gamma(\delta, \gamma, \lambda, \mathfrak{s}, \mathfrak{t}, q))-h_{3}(r) \mathrm{Y}(\delta, \gamma, \lambda, \mathfrak{s}, \mathfrak{t}, q)\right]},
$$

where $\Omega(\delta, \gamma, \lambda, \mathfrak{s}, \mathfrak{t}, q), \Gamma(\delta, \gamma, \lambda, \mathfrak{s}, \mathfrak{t}, q)$ and $Y(\delta, \gamma, \lambda, \mathfrak{s}, \mathfrak{t}, q)$ are given by (7)-(9), respectively.

By further computations using (5), (16) and (24), we obtain

$$
\begin{gathered}
\left|a_{2}\right| \leqq \frac{|b r|[2+\mathfrak{t}]_{\mathfrak{q}}^{\mathfrak{s}} \sqrt{|b r|[3+\mathfrak{t}]_{\mathfrak{q}}^{\mathfrak{s}}}}{\Lambda(\delta, \gamma, \lambda, \mathfrak{s}, \mathfrak{t}, q ; b . r)} \\
(\Lambda(\delta, \gamma, \lambda, \mathfrak{s}, \mathfrak{t}, q ; b . r) \\
\left.:=\sqrt{\left|[(\Omega(\delta, \gamma, \lambda, \mathfrak{s}, \mathfrak{t}, q)+\Gamma(\delta, \gamma, \lambda, \mathfrak{s}, \mathfrak{t}, q)) b-\mathfrak{p Y}(\delta, \gamma, \lambda, \mathfrak{s}, \mathfrak{t}, q)] b r^{2}-\mathfrak{q} a Y(\delta, \gamma, \lambda, \mathfrak{s}, \mathfrak{t}, q)\right|}\right) .
\end{gathered}
$$

Next, if we subtract (20) from (18), we can easily see that

$$
\begin{gathered}
\frac{2[(1-\delta)(\gamma+2)+\delta(3 \lambda-1)][1+\mathfrak{t}]_{\mathfrak{q}}^{\mathfrak{s}}}{[3+\mathfrak{t}]_{\mathfrak{q}}^{\mathfrak{F}}}\left(a_{3}-a_{2}^{2}\right) \\
=h_{2}(r)\left(u_{2}-v_{2}\right)+h_{3}(r)\left(u_{1}^{2}-v_{1}^{2}\right) .
\end{gathered}
$$

In the light of (21) and (22), we conclude from (25) that

$$
\begin{aligned}
a_{3}=\frac{h_{2}(r)[3+\mathfrak{t}]_{\mathfrak{q}}^{\mathfrak{s}}\left(u_{2}-v_{2}\right)}{2[(1-\delta)(\gamma+2)+\delta(3 \lambda-1)][1+\mathfrak{t}]_{\mathfrak{q}}^{\mathfrak{s}}} & \\
& \quad+\frac{h_{2}^{2}(r)[2+\mathfrak{t}]_{q}^{2 \mathfrak{s}}\left(u_{1}^{2}+v_{1}^{2}\right)}{2[(1-\delta)(\gamma+1)+\delta(2 \lambda-1)]^{2}[1+\mathfrak{t}]_{\mathfrak{q}}^{2 \mathfrak{s}}} .
\end{aligned}
$$

Thus, by applying (5), we obtain the following inequality:

$$
\begin{aligned}
\left|a_{3}\right| \leqq \frac{|b r|[3+\mathfrak{t}]_{q}^{\mathfrak{s}}}{[(1-\delta)(\gamma+2)+\delta(3 \lambda-1)][1+\mathfrak{t}]_{\mathfrak{q}}^{\mathfrak{s}}} & \\
& \quad+\frac{b^{2} r^{2}[2+\mathfrak{t}]_{q}^{2 \mathfrak{s}}}{[(1-\delta)(\gamma+1)+\delta(2 \lambda-1)]^{2}[1+\mathfrak{t}]_{\mathfrak{q}}^{2 \mathfrak{s}}} .
\end{aligned}
$$


This completes the proof of Theorem 1.

In the next theorem, we present the Fekete-Szegö inequality for $\mathcal{S W}_{\Sigma}(\delta, \gamma, \lambda, \mathfrak{s , t}, q, r)$.

Theorem 2. For $0 \leqq \delta \leqq 1, \gamma \geqq 0, \lambda \geqq 1$ and $r, \mu \in \mathbb{R}$, let $f \in \mathcal{A}$ be in the family $\mathcal{S W}_{\Sigma}(\delta, \gamma, \lambda, \mathfrak{s}, \mathfrak{t}, q, r)$. Then

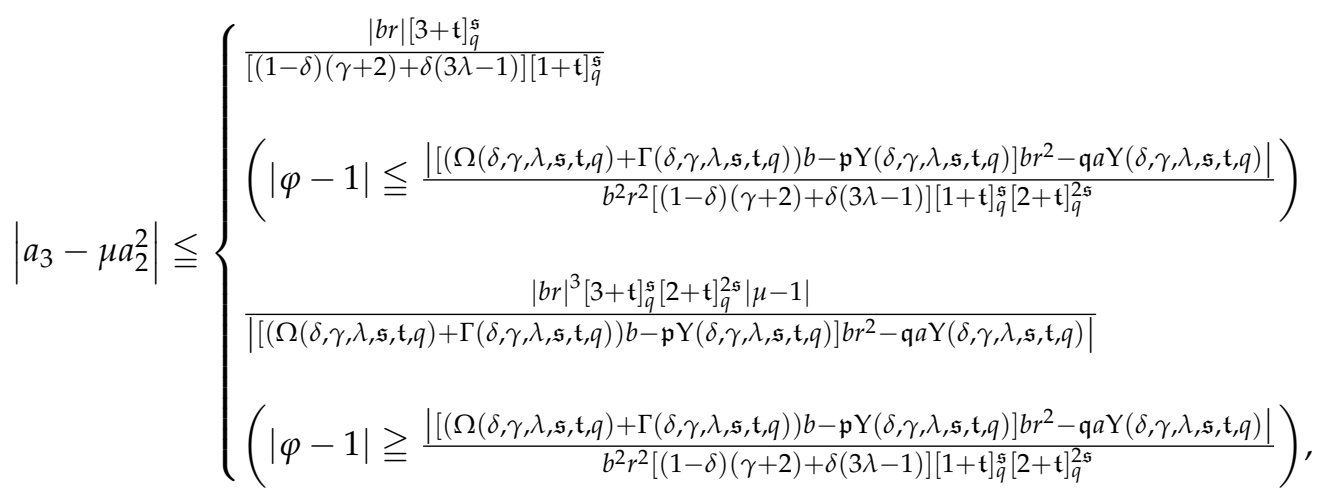

where, for convenience,

$$
\varphi=\varphi(\mu, r):=\frac{h_{2}^{2}(r)[3+\mathfrak{t}]_{q}^{\mathfrak{s}}[2+\mathfrak{t}]_{q}^{2 \mathfrak{s}}(1-\mu)}{h_{2}^{2}(r)(\Omega(\delta, \gamma, \lambda, \mathfrak{s}, \mathfrak{t}, q)+\Gamma(\delta, \gamma, \lambda, \mathfrak{s}, \mathfrak{t}, q))-h_{3}(r) \mathrm{Y}(\delta, \gamma, \lambda, \mathfrak{s}, \mathfrak{t}, q)} .
$$

Proof. It follows from (24) and (25) that

$$
\begin{aligned}
a_{3}-\mu a_{2}^{2}= & \frac{h_{2}(r)[3+\mathfrak{t}]_{q}^{\mathfrak{s}}\left(u_{2}-v_{2}\right)}{2[(1-\delta)(\gamma+2)+\delta(3 \lambda-1)][1+\mathfrak{t}]_{q}^{\mathfrak{s}}}+(1-\mu) a_{2}^{2} \\
= & \frac{h_{2}(r)[3+\mathfrak{t}]_{q}^{\mathfrak{F}}\left(u_{2}-v_{2}\right)}{2[(1-\delta)(\gamma+2)+\delta(3 \lambda-1)][1+\mathfrak{t}]_{\mathfrak{q}}^{\mathfrak{s}}} \\
& \quad+\frac{h_{2}^{3}(r)[3+\mathfrak{t}]_{q}^{\mathfrak{s}}[2+\mathfrak{t}]_{q}^{2 \mathfrak{s}}\left(u_{2}+v_{2}\right)(1-\mu)}{2\left[h_{2}^{2}(r)(\Omega(\delta, \gamma, \lambda, \mathfrak{s}, \mathfrak{t}, q)+\Gamma(\delta, \gamma, \lambda, \mathfrak{s}, \mathfrak{t}, q))-h_{3}(r) \mathrm{Y}(\delta, \gamma, \lambda, \mathfrak{s}, \mathfrak{t}, q)\right]} \\
= & \frac{h_{2}(r)}{2}\left[\left(\varphi(\mu, r)+\frac{[3+\mathfrak{t}]_{q}^{\mathfrak{s}}}{[(1-\delta)(\gamma+2)+\delta(3 \lambda-1)][1+\mathfrak{t}]_{\mathfrak{q}}^{\mathfrak{s}}}\right) u_{2}\right. \\
& \left.\quad+\left(\varphi(\mu, r)-\frac{[3+\mathfrak{t}]_{q}^{\mathfrak{s}}}{[(1-\delta)(\gamma+2)+\delta(3 \lambda-1)][1+\mathfrak{t}]_{\mathfrak{q}}^{\mathfrak{s}}}\right) v_{2}\right]
\end{aligned}
$$

where, just as stated in Theorem 2,

$$
\varphi(\mu, r)=\frac{h_{2}^{2}(r)[3+\mathfrak{t}]_{q}^{\mathfrak{s}}[2+\mathfrak{t}]_{q}^{2 \mathfrak{s}}(1-\mu)}{h_{2}^{2}(r)(\Omega(\delta, \gamma, \lambda, \mathfrak{s}, \mathfrak{t}, q)+\Gamma(\delta, \gamma, \lambda, \mathfrak{s}, \mathfrak{t}, q))-h_{3}(r) \mathrm{Y}(\delta, \gamma, \lambda, \mathfrak{s}, \mathfrak{t}, q)} .
$$


Thus, according to (5), we have the following inequality:

$$
\left|a_{3}-\mu a_{2}^{2}\right| \leqq\left\{\begin{array}{l}
\frac{|b r|[3+\mathfrak{t}]_{q}^{\mathfrak{s}}}{[(1-\delta)(\gamma+2)+\delta(3 \lambda-1)][1+\mathfrak{t}]_{q}^{\mathfrak{5}}} \\
\left(0 \leqq|\varphi(\mu, r)| \leqq \frac{[3+\mathfrak{t}]_{q}^{\mathfrak{s}}}{[(1-\delta)(\gamma+2)+\delta(3 \lambda-1)][1+\mathfrak{t}]_{q}^{5}}\right) \\
|b r| \cdot|\varphi(\mu, r)| \\
\left(|\varphi(\mu, r)| \geqq \frac{[3+\mathfrak{t}]_{q}^{\mathfrak{s}}}{\left.[(1-\delta)(\gamma+2)+\delta(3 \lambda-1)][1+\mathfrak{t}]_{9}^{5}\right)}\right.
\end{array}\right.
$$

which, after simple computation, yields the following inequality:

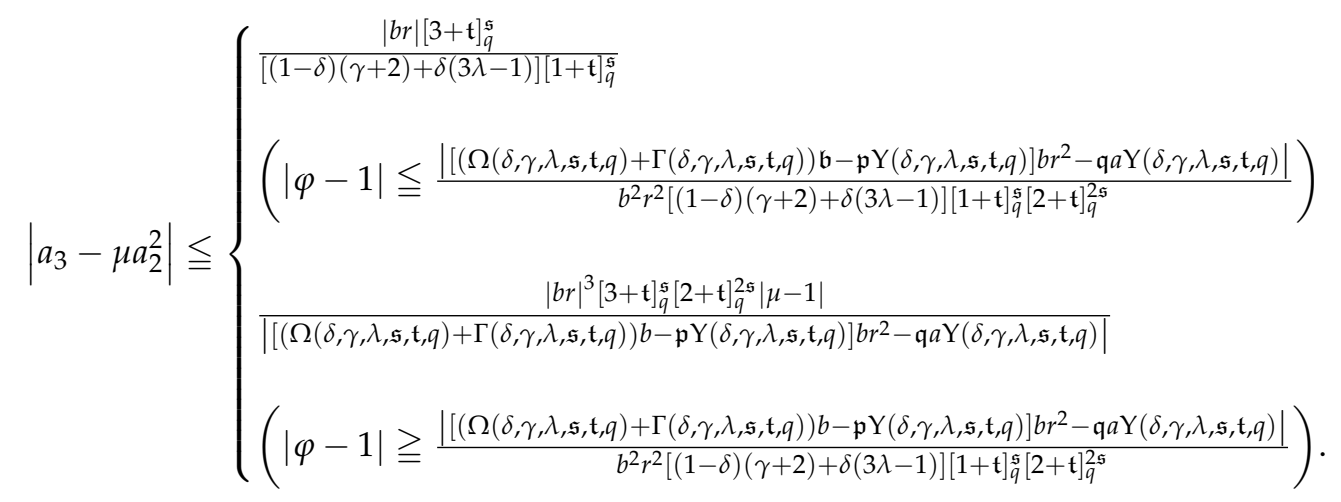

We have thus completed the proof of Theorem 2.

\section{Special Cases and Consequences}

In this section, we choose to specialize our main results asserted by Theorem 1 and Theorem 2.

By putting $\mu=1$ in Theorem 2, we are led to the following corollary.

Corollary 1. For $0 \leqq \delta \leqq 1, \gamma \geqq 0, \lambda \geqq 1$ and $r \in \mathbb{R}$, let $f \in \mathcal{A}$ be in the family $\mathcal{S W}_{\Sigma}(\delta, \gamma, \lambda, \mathfrak{s}, \mathfrak{t}, q, r)$. Then

$$
\left|a_{3}-a_{2}^{2}\right| \leqq \frac{|b r|[3+\mathfrak{t}]_{q}^{\mathfrak{s}}}{[(1-\delta)(\gamma+2)+\delta(3 \lambda-1)][1+\mathfrak{t}]_{\mathfrak{q}}^{\mathfrak{g}}} .
$$

Remark 5. The family $\mathcal{S} \mathcal{W}_{\Sigma}(\delta, \gamma, \lambda, \mathfrak{s}, \mathfrak{t}, q, r)$ generalizes several known families of bi-univalent functions. We list them as follows.

1. For $\mathfrak{s}=\delta=0$, we have

$$
\mathcal{S W}_{\Sigma}(\delta, \gamma, \lambda, \mathfrak{s}, \mathfrak{t}, q, r)=: \mathcal{N}_{\Sigma}(\gamma, r),
$$

where $\mathcal{N}_{\Sigma}(\gamma, r)$ is the bi-univalent function family studied recently by Wanas and Lupas [54].

2. For $\mathfrak{s}=\delta=\gamma=0$, we have

$$
\mathcal{S W}_{\Sigma}(\delta, \gamma, \lambda, \mathfrak{s}, \mathfrak{t}, q, r)=: S_{\Sigma}^{*}(r),
$$

where $S_{\Sigma}^{*}(r)$ denote the bi-univalent function family studied by Srivastava et al. [47]. 
3. For $\mathfrak{s}=\delta=0$ and $\gamma=1$, we have the following relationship:

$$
\mathcal{S} \mathcal{W}_{\Sigma}(\delta, \gamma, \lambda, \mathfrak{s}, \mathfrak{t}, q, r)=: \Sigma^{\prime}(r),
$$

where $\Sigma^{\prime}(r)$ is the bi-univalent function family introduced by Alamoush [49].

4. For $\mathfrak{s}=\delta=0, a=1, b=\mathfrak{p}=2, \mathfrak{q}=-1$ and $r \longrightarrow t$, we have

$$
\mathcal{S W}_{\Sigma}(\delta, \gamma, \lambda, \mathfrak{s}, \mathfrak{t}, q, r)=: \mathcal{B}_{\Sigma}^{\gamma}(t),
$$

where $\mathcal{B}_{\Sigma}^{\gamma}(t)$ is the bi-univalent function family introduced by Bulut et al. [55].

5. For $\mathfrak{s}=0, \delta=a=1, b=\mathfrak{p}=2, \mathfrak{q}=-1$ and $r \longrightarrow t$, we have

$$
\mathcal{S W}_{\Sigma}(\delta, \gamma, \lambda, \mathfrak{s}, \mathfrak{t}, q, r)=: \mathcal{L B}_{\Sigma}(\lambda, t)
$$

where $\mathcal{L B}_{\Sigma}(\lambda, t)$ is the bi-univalent function family investigated by Magesh and Bulut [56].

6. For $\mathfrak{s}=\delta=\gamma=0, a=1, b=\mathfrak{p}=2, \mathfrak{q}=-1$ and $r \longrightarrow t$, we have

$$
\mathcal{S} \mathcal{W}_{\Sigma}(\delta, \gamma, \lambda, \mathfrak{s}, \mathfrak{t}, q, r)=: S_{\Sigma}(t),
$$

where $S_{\Sigma}(t)$ is the bi-univalent function family given by Altınkaya and Yalçin [57].

7. For $\mathfrak{s}=\delta=0, \gamma=a=1, b=\mathfrak{p}=2, \mathfrak{q}=-1$ and $r \longrightarrow t$, we have

$$
\mathcal{S W}_{\Sigma}(\delta, \gamma, \lambda, \mathfrak{s}, \mathfrak{t}, q, r)=: \mathcal{B}_{\Sigma}(t),
$$

where $\mathcal{B}_{\Sigma}(t)$ is the bi-univalent function family given by Bulut et al. [55].

8. For $\mathfrak{s}=\delta=0, a=1, b=\mathfrak{p}=2, \mathfrak{q}=-1, r \longrightarrow t$ and

$$
\Pi(t, z)=\left(\frac{1}{1-2 t z+z^{2}}\right)^{\alpha} \quad(0<\alpha \leqq 1),
$$

we have

$$
\mathcal{S W}_{\Sigma}(\delta, \gamma, \lambda, \mathfrak{s}, \mathfrak{t}, q, r)=: P_{\Sigma}(\alpha, \gamma)
$$

where $P_{\Sigma}(\alpha, \gamma)$ is the bi-univalent function family considered by Prema and Keerthi [58].

9. For $\mathfrak{s}=0, \delta=a=1, b=\mathfrak{p}=2, \mathfrak{q}=-1, r \longrightarrow t$ and

$$
\Pi(t, z)=\left(\frac{1}{1-2 t z+z^{2}}\right)^{\alpha} \quad(0<\alpha \leqq 1),
$$

we have

$$
\mathcal{S} \mathcal{W}_{\Sigma}(\delta, \gamma, \lambda, \mathfrak{s}, \mathfrak{t}, q, r)=: \mathfrak{L} B_{\Sigma}^{\lambda}(\alpha),
$$

where $\mathfrak{L} B_{\Sigma}^{\lambda}(\alpha)$ is the bi-univalent function family considered by Joshi et al. [59].

10. For $\mathfrak{s}=\delta=\gamma=0, a=1, b=\mathfrak{p}=2, \mathfrak{q}=-1, r \longrightarrow t$ and

$$
\Pi(t, z)=\left(\frac{1}{1-2 t z+z^{2}}\right)^{\alpha} \quad(0<\alpha \leqq 1),
$$

we have

$$
\mathcal{S W}_{\Sigma}(\delta, \gamma, \lambda, \mathfrak{s}, \mathfrak{t}, q, r)=: S_{\Sigma}^{*}(\alpha),
$$

where $S_{\Sigma}^{*}(\alpha)$ is the bi-univalent function family introduced by Brannan and Taha [60].

11. For $\mathfrak{s}=\delta=0, \gamma=a=1, b=\mathfrak{p}=2, \mathfrak{q}=-1, r \longrightarrow t$ and

$$
\Pi(t, z)=\left(\frac{1}{1-2 t z+z^{2}}\right)^{\alpha} \quad(0<\alpha \leqq 1),
$$


we have

$$
\mathcal{S W}_{\Sigma}(\delta, \gamma, \lambda, \mathfrak{s}, \mathfrak{t}, q, r)=: \mathcal{H}_{\Sigma}^{\alpha}
$$

where $\mathcal{H}_{\Sigma}^{\alpha}$ is the bi-univalent function family considered by Srivastava et al. [2].

Remark 6. For particular choices of $\mathfrak{s}, \delta, \gamma, a, b, \mathfrak{p}$ and $\mathfrak{q}$, Theorem 1 and Theorem 2 reduce to $a$ number of known results, which are given below.

1. If we put $\mathfrak{s}=\delta=0$ in our Theorems, we have the corresponding results for wellknown family $\mathcal{N}_{\Sigma}(\gamma, r)$ of bi-Bazilevič functions which was studied recently by Wanas and Lupas [54].

2. If we put $\mathfrak{s}=\delta=\gamma=0$ in our Theorems, we have the corresponding results for the family $S_{\Sigma}^{*}(r)$, which was considered recently by Srivastava et al. [47].

3. If we put $\mathfrak{s}=\delta=0$ and $\gamma=1$ in our Theorems, we have the corresponding results for the known family $\Sigma^{\prime}(r)$, which was studied recently by Al-Amoush [49].

4. If we put $\mathfrak{s}=\delta=0, a=1, b=\mathfrak{p}=2, \mathfrak{q}=-1$ and $r \longrightarrow t$ in our Theorems, we have the corresponding results for the family of $\mathcal{B}_{\Sigma}^{\gamma}(t)$ of bi-Bazilevič functions, which was discussed recently by Bulut et al. [55].

5. If we put $\mathfrak{s}=0, \delta=a=1, b=\mathfrak{p}=2, \mathfrak{q}=-1$ and $r \longrightarrow t$ in our Theorems, we have the corresponding results for the family $\mathcal{L B}_{\Sigma}(\lambda, t)$ of bi-pseudo-starlike functions, which was studied recently by Magesh and Bulut [56].

6. If we put $\mathfrak{s}=\delta=\gamma=0, a=1, b=\mathfrak{p}=2, \mathfrak{q}=-1$ and $r \longrightarrow t$ in our Theorems, we obtain the corresponding results for the family $S_{\Sigma}(t)$ of bi-starlike functions, which was considered recently by Altınkaya and Yalçin [57].

7. If we put $\mathfrak{s}=\delta=0, \gamma=a=1, b=\mathfrak{p}=2, \mathfrak{q}=-1$ and $r \longrightarrow t$ in our Theorems, we obtain the corresponding results for the family $\mathcal{B}_{\Sigma}(t)$ which was discussed recently by Bulut et al. [55].

\section{Conclusions}

The fact that we can find many unique and effective usages of a large variety of interesting special functions and specific polynomials in Geometric Function Theory of Complex Analysis provided the primary inspiration and motivation for our analysis in this article. Our main objective was to create a new family $\mathcal{S} \mathcal{W}_{\Sigma}(\delta, \gamma, \lambda, \mathfrak{s , t}, q, r)$ of holomorphic and bi-univalent functions, which is defined by means of the $q$-Srivastava-Attiya operator and by also using the Horadam polynomial $h_{n}(r)$ given by the recurrence relation (5) and by generating function $\Pi(r, z)$ in (6). We derived inequalities for the initial Taylor-Maclaurin coefficients of functions belonging to this newly-introduced holomorphic and bi-univalent function class $\mathcal{S} \mathcal{W}_{\Sigma}(\delta, \gamma, \lambda, \mathfrak{s}, \mathfrak{t}, q, r)$. Furthermore, we investigated the celebrated FeketeSzegö problem for this general holomorphic and bi-univalent function class. We also pointed out several important correlations between our findings and those which were considered in previous studies.

We remark further that, since the additional parameter $p$ is obviously superfluous, Srivastava ([34], p. 340) exposed the so-called $(p, q)$-calculus as a rather trivial and inconsequential variation of the classical $q$-calculus. So, clearly, while we do encourage and support the $q$-results of the kind which we have presented in this paper as well as potential $q$-extensions of other analogous developments in Applicable Mathematical Analysis, we do not encourage and support the so-called $(p, q)$-variations of the suggested $q$-results by inconsequentially and trivially adding a redundant parameter $p$.

Author Contributions: Conceptualization, R.S., A.K.W. and H.M.S.; methodology, A.K.W., R.S. and H.M.S.; software, A.K.W.; validation, H.M.S., R.S. and A.K.W.; formal analysis, A.K.W. and H.M.S.; investigation, H.M.S. and A.K.W.; resources, H.M.S.; data curation, A.K.W. and H.M.S.; writingoriginal draft preparation, A.K.W.; writing-review and editing, R.S. and H.M.S.; visualization, H.M.S. and A.K.W.; supervision, H.M.S.; project administration, R.S.; funding acquisition, R.S. All authors have read and agreed to the published version of the manuscript. 
Funding: This research received no external funding.

Institutional Review Board Statement: Not applicable.

Informed Consent Statement: Not applicable.

Data Availability Statement: Not applicable.

Conflicts of Interest: The authors declare no conflict of interest.

\section{References}

1. Duren, P.L. Univalent Functions; Grundlehren der Mathematischen Wissenschaften, Band 259; Springer: New York, NY, USA; Berlin/Heidelberg, Germany; Tokyo, Japan, 1983.

2. Srivastava, H.M.; Mishra, A.K.; Gochhayat, P. Certain subclasses of analytic and bi-univalent functions. Appl. Math. Lett. 2010, 23, 1188-1192. [CrossRef]

3. Bulut, S. Coefficient estimates for general subclasses of $m$-fold symmetric analytic bi-univalent functions. Turkish J. Math. 2016, 40, 1386-1397. [CrossRef]

4. Bulut, S.; Wanas, A.K. Coefficient estimates for families of bi-univalent functions defined by Ruscheweyh derivative operator. Math. Moravica 2021, 25, 71-80. [CrossRef]

5. Güney, H.Ö.; Murugusundaramoorthy, G.; Sokół, J. Subclasses of bi-univalent functions related to shell-like curves connected with Fibonacci numbers. Acta Univ. Sapient. Math. 2018, 10, 70-84. [CrossRef]

6. Páll-Szabó, Á.O.; Wanas, A.K. Coefficient estimates for some new classes of bi-Bazilevič functions of Ma-Minda type involving the Sălăgean integro-differential operator. Quaest. Math. 2021, 44, 495-502.

7. Srivastava, H.M.; Wanas, A.K. Initial Maclaurin coefficient bounds for new subclasses of analytic and $m$-fold symmetric biunivalent functions defined by a linear combination. Kyungpook Math. J. 2019, 59, 493-503.

8. Fekete, M.; Szegö, G. Eine bemerkung uber ungerade schlichte funktionen. J. Lond. Math. Soc. 1933, 2, 85-89. [CrossRef]

9. Srivastava, H.M.; Mishra, A.K.; Das, M.K. The Fekete-Szegö problem for a subclass of close-to-convex functions. Complex Variables Theory Appl. 2001, 44, 145-163. [CrossRef]

10. Srivastava, H.M.; Raza, N.; AbuJarad, E.S.A.; AbuJarad, G.S.M.H. Fekete-Szegö inequality for classes of $(p, q)$-starlike and $(p, q)$-convex functions. Rev. Real Acad. Cienc. Exactas Fís. Natur. Ser. A Mat. 2019, 113, 3563-3584. [CrossRef]

11. Singh, R. On Bazilevič functions. Proc. Amer. Math. Soc. 1973, 38, 261-271.

12. Babalola, K.O. On $\lambda$-Pseudo-starlike functions. J. Class. Anal. 2013, 3, 137-147. [CrossRef]

13. Miller, S.S.; Mocanu, P.T. Differential Subordinations: Theory and Applications; Series on Monographs and Textbooks in Pure and Applied Mathematics; Marcel Dekker Incorporated: New York, NY, USA, 2000; Volume 225.

14. Jackson, F.H. On $q$-functions and a certain difference operator. Trans. Royal Soc. Edinburgh 1908, 46, 253-281. [CrossRef]

15. Jackson, F.H. On q-definite integrals. Quart. J. Pure Appl. Math. 1910, 41, 193-203.

16. Exton, H. q-Hypergeometric Functions and Applications; Ellis Horwood Limited: Chichester, UK, 1983.

17. Gasper, G.; Rahman, M. Basic Hypergeometric Series, 2nd ed.; Encyclopedia of Mathematics and Its Applications; Cambridge University Press: Cambridge, MA, USA, 2004; Volume 96.

18. Ghany, H.A. q-Derivative of basic hypergeomtric series with respect to parameters. Internat. J. Math. Anal. 2009, 3, 1617-1632.

19. Srivastava, H.M.; Attiya, A.A. An integral operator associated with the Hurwitz-Lerch Zeta function and differential subordination. Integral Transform. Spec. Funct. 2007, 18, 207-216. [CrossRef]

20. Srivastava, H.M. The Zeta and related functions: Recent developments. J. Adv. Engrg. Comput. 2019, 3, 329-354. [CrossRef]

21. Srivastava, H.M. Some general families of the Hurwitz-Lerch Zeta functions and their applications: Recent developments and directions for further researches. Proc. Inst. Math. Mech. Nat. Acad. Sci. Azerbaijan 2019, 45, 234-269. [CrossRef]

22. Erdélyi, A.; Magnus, W.; Oberhettinger, F.; Tricomi, F.G. Higher Transcendental Functions; McGraw-Hill Book Company: New York, NY, USA, 1953; Volume I.

23. Shah, S.A.; Noor, K.I. Study on the $q$-analogue of a certain family of linear operators. Turkish J. Math. 2019, 43, 2707-2714. [CrossRef]

24. Deniz, E.; Kamali, M.; Korkmaz, S. A certain subclass of bi-univalent functions associated with Bell numbers and $q$-Srivastava Attiya operator. AIMS Math. 2020, 5, 7259-7271. [CrossRef]

25. Altıntaş, O.; Mustafa, N. Coefficient bounds and distortion theorems for the certain analytic functions. Turkish J. Math. 2019, 43, 985-997. [CrossRef]

26. Kanas, S.; Răducanu, D. Some class of analytic functions related to conic domains. Math. Slovaca 2014, 64, 1183-1196. [CrossRef]

27. Khan, Q.; Arif, M.; Raza, M.; Srivastava, G.; Tang, H. Some applications of a new integral operator in $q$-analog for multivalent functions. Mathematics 2019, 7, 1178. [CrossRef]

28. Khan, B.; Srivastava, H.M.; Tahir, M.; Darus, M.; Ahmad, Q.Z.; Khan, N. Applications of a certain $q$-integral operator to the subclasses of analytic and bi-univalent functions. AIMS Math. 2021, 6, 1024-1039. [CrossRef]

29. Mahmood, S.; Raza, N.; Abujarad, E.S.A.; Srivastava, G.; Srivastava, H.M.; Malik, S.N. Geometric properties of certain classes of analytic functions associated with a $q$-integral operator. Symmetry 2019, 11, 719. [CrossRef]

30. Noor, K.I. On generalized q-close-to-convexity. Appl. Math. Inform. Sci. 2017, 11, 1383-1388. [CrossRef] 
31. Noor, K.I.; Riaz, S. Generalized q-starlike functions. Stud. Sci. Math. Hungar. 2017, 54, 509-522. [CrossRef]

32. Shamsan, H.; Latha, S. On generalized bounded Mocanu variation related to $q$-derivative and conic regions. Ann. Pure Appl. Math. 2018, 17, 67-83. [CrossRef]

33. Shi, L.; Khan, Q.; Srivastava, G.; Liu, J.-L.; Arif, M. A study of multivalent $q$-starlike functions connected with circular domain. Mathematics 2019, 7, 670. [CrossRef]

34. Srivastava, H.M. Operators of basic (or $q$-) calculus and fractional $q$-calculus and their applications in geometric function theory of complex analysis. Iran. J. Sci. Technol. Trans. A Sci. 2020, 44, 327-344. [CrossRef]

35. Kac, V.; Cheung, P. Symmetric quantum calculus. In Quantum Calculus; Universitext Book Series; Springer: Berlin/Heidelberg, Germany, 2002; pp. 99-104.

36. Srivastava, H.M.; Juma, A.S.; Zayed, H.M. Univalence conditions for an integral operator defined by a generalization of the Srivastava-Attiya operator. Filomat 2018, 32, 2101-2114. [CrossRef]

37. Sim, Y.J.; Kwon, O.S.; Cho, N.E.; Srivastava, H.M. Bounds for the real parts and arguments of normalized analytic functions defined by the Srivastava-Attiya operator. J. Comput. Anal. Appl. 2020, 28, 628-645.

38. Srivastava, H.M.; Prajapati, A.; Gochhayat, P. Third-order differential subordination and differential superordination results for analytic functions involving the Srivastava-Attiya operator. Appl. Math. Inform. Sci. 2018, 12, 469-481. [CrossRef]

39. Noor, K.I.; Riaz, S.; Noor, M.A. On q-Bernardi integral operator. TWMS J. Pure Appl. Math. 2017, 8, 3-11.

40. Bernardi, S.D. Convex and starlike univalent functions. Trans. Amer. Math. Soc. 1969, 135, 429-446. [CrossRef]

41. Alexander, J.W. Functions which map the interior of the unit circle upon simple region. Ann. Math. 1915, 17, 12-22. [CrossRef]

42. Hörçum, T.; Koçer, E.G. On some properties of Horadam polynomials. Internat. Math. Forum. 2009, 4, $1243-1252$.

43. Horadam, A.F.; Mahon, J.M. Pell and Pell-Lucas polynomials. Fibonacci Quart. 1985, 23, 7-20.

44. Horadam, A.F. Jacobsthal representation polynomials. Fibonacci Quart. 1997, 35, 137-148.

45. Koshy, T. Fibonacci and Lucas Numbers with Applications; John Wiley and Sons: New York, NY, USA, 2001.

46. Lupas, A. A guide of Fibonacci and Lucas polynomials. Octagon Math. Mag. 1999, 7, 2-12.

47. Srivastava, H.M.; Altınkaya, Ş.; Yalçin, S. Certain subclasses of bi-univalent functions associated with the Horadam polynomials. Iran. J. Sci. Technol. Trans. A Sci. 2019, 43, 1873-1879. [CrossRef]

48. Abirami, C.; Magesh, N.; Yamini, J. Initial bounds for certain classes of bi-univalent functions defined by Horadam polynomials. Abstr. Appl. Anal. 2020, 2020, 7391058. [CrossRef]

49. Al-Amoush, A.G. Coefficient estimates for certain subclass of bi functions associated with the Horadam polynomials. arXiv 2018, arXiv:1812.10589v1.

50. Al-Amoush, A.G. Certain subclasses of bi-univalent functions involving the Poisson distribution associated with Horadam polynomials. Malaya J. Mat. 2019, 7, 618-624. [CrossRef]

51. Al-Amoush, A.G. Coefficient estimates for a new subclasses of $\lambda$-pseudo biunivalent functions with respect to symmetrical points associated with the Horadam polynomials. Turkish J. Math. 2019, 43, 2865-2875. [CrossRef]

52. Srivastava, H.M.; Wanas, A.K.; Murugusundaramoorthy, G. Certain family of bi-univalent functions associated with Pascal distribution series based on Horadam polynomials. Surveys Math. Appl. 2021, 16, 193-205.

53. Wanas, A.K.; Yalçin, S. Horadam polynomials and their applications to new family of bi-univalent functions with respect to symmetric conjugate points. Proyecciones J. Math. 2021, 40, 107-116. [CrossRef]

54. Wanas, A.K.; Lupas, A.A. Applications of Horadam polynomials on Bazilevič bi-univalent function satisfying subordinate conditions. J. Phys. Conf. Ser. 2019, 1294, 1-6.

55. Bulut, S.; Magesh, N.; Abirami, C. A comprehensive class of analytic bi-univalent functions by means of Chebyshev polynomials. J. Fract. Calc. Appl. 2017, 8, 32-39.

56. Magesh, N.; Bulut, S. Chebyshev polynomial coefficient estimates for a class of analytic bi-univalent functions related to pseudo-starlike functions. Afrika Mat. 2018, 29, 203-209. [CrossRef]

57. Altınkaya, Ş.; Yalçin, S. On the Chebyshev polynomial coefficient problem of some subclasses of bi-univalent functions. Gulf J. Math. 2017, 5, 34-40.

58. Prema, S.; Keerthi, B.S. Coefficient bounds for certain subclasses of analytic function. J. Math. Anal. 2013, 4, $22-27$.

59. Joshi, S.; Joshi, S.; Pawar, H. On some subclasses of bi-univalent functions associated with pseudo-starlike functions. J. Egypt. Math. Soc. 2016, 24, 522-525. [CrossRef]

60. Brannan, D.A.; Taha, T.S. On some classes of bi-univalent functions. Studia Univ. Babeş-Bolyai Math. 1986, 31, 70-77. 